\title{
Acknowledgement to Reviewers of IJGI in 2016
}

\author{
IJGI Editorial Office \\ Published: 11 January 2017 \\ MDPI AG, St. Alban-Anlage 66, 4052 Basel, Switzerland; ijgi@mdpi.com
}

The editors of IJGI would like to express their sincere gratitude to the following reviewers for assessing manuscripts in 2016.

We greatly appreciate the contribution of expert reviewers, which is crucial to the journal's editorial process. We aim to recognize reviewer contributions through several mechanisms, of which the annual publication of reviewer names is one. Reviewers receive a voucher entitling them to a discount on their next MDPI publication and can download a certificate of recognition directly from our submission system. Additionally, reviewers can sign up to the service Publons (https://publons.com) to receive recognition. Of course, in these intiatives we are careful not to compromise reviewer confidentiality. Many reviewers see their work as a voluntary and often unseen part of their role as researchers. We are grateful to the time reviewers donate to our journals and the contribution they make.

If you are interested in becoming a reviewer for IJGI, see the link at the bottom of the webpage http://www.mdpi.com/reviewers.

The following reviewed for IJGI in 2016:

Abarca-Hernandez, F. Abouali, Mohammad Acedo, Luis

Agam, Gady

Agapiou, Athos

AghaKouchak, Amir

Aguirre-Gutiérrez, Jesús

Ahlers, Dirk

Ahmed, Sohel J.

Ahn, Yushin

Akleman, Ergun

Alexander, David

Al-Hussein, Abdullah

Ali, Ahmed Loai

Ali, Muhammad Intizar

Alim, Samat

Allasia, Paolo

Al-Masri, Eyhab

Alonso, Jose Juan

Alshurafa, Nabil

Al-Yaari, Amen

Amirian, Pouria

Amirpour Haredasht, Sara

Amit, Kulkarni

Amitrano, Donato

Amram, Ofer
Anderson, Sharolyn

Andreas, Koch

Andris, Clio

Antolini, Francesco

Antonopoulos, Angelos

Aplin, Paul

Arcidiacono, Claudia

Ardizzone, Francesca

Aretano, Roberta

Argyriou, Athanasios V.

Ariyur, Kartik

Ariza, Francisco

Arroyo Ohori, Ken

Artese, Giuseppe

Asorey-Cacheda, Rafael

Atia, Mohamed

Auch, Roger

Ayuga, Francisco

Badreldin, Nasem

Bae, Kwang-Ho

Baiocchi, Valerio

Bakalov, Petko

Bakuła, Krzysztof

Baležentis, Tomas

Barb, Adrian

Barlow, Nadine

\author{
Barrera Rosillo, Domingo \\ Barsi, Arpad \\ Bartoněk, Dalibor \\ Bathrellos, George D. \\ Baumann, Peter \\ Bedogni, Luca \\ Belgiawan, Prawira \\ Fajarindra \\ Belgiu, Mariana \\ Bell, Scott \\ Bellido, Francisco J. \\ Benedict, Karl \\ Benitez, Francisco Garcia \\ Beongku, An \\ Bereuter, Pia \\ Berland, Adam \\ Bernabé, Sergio \\ Bernhard, Luzi \\ Beyer, Hans-georg \\ Bhandari, Santosh \\ Bhandari, Subodh \\ Bhattarai, Keshav \\ Bigelow, Daniel \\ Bill, Ralf \\ Bischoff, Joschka \\ Bisegna, Fabio
}


Bitelli, Gabriele

Black, John

Blanco, Julio

Blesius, Leonhard

Blomqvist, Eva

Bordogna, Gloria

Borzacchiello, Maria Teresa

Both, Alan

Boulmakoul, Azedine

Brando, Carmen

Brandt, Scott

Brasebin, Mickael

Brisco, Brian

Brodaric, Boyan

Brodeur, Jean

Bronisz, Urszula

Brychtova, Alzbeta

Bucała-Hrabia, Anna

Buccella, Agustina

Buenemann, Michaela

Cabral, Pedro

Cahyadi, Mokhamad Nur

Calbimonte, Jean-Paul

Callaghan, Sarah

Callaghan, Sarah

Camara, Carmen

Camarda, Domenico

Cameron, Michael

Campagna, Michele

Cannelle, Bertrand

Cao, Kai

Cao, Yang

Caponetti, Laura

Carlson, Toby

Casado, Rubén

Casas, Irene

Caselles, Vicente

Cawkwell, Fiona

Cerreta, Maria

Chan, Chiu-shui

Chan, Pak Wai

Chandrasekar, V.

Chang, Jiyul

Chang, Yu-Chia

Chau, K.W.

Chavoshi, Seyed Hossein

CHE, Weitao

Chen, Biyu

Chen, Chih-Yuan

Chen, Kongzhe

Chen, Nengcheng

Chen, Ruey-Shun
Chen, ung-Hui

Chen, Wenyu

Chen, Yen-Lun

Chen, Yumin

Cheng, Changxiu

Cheng, Tao

Cheung, Darren Man-wai

Chiabrando, F.

Chiang, Kai-Wei

Chiang, Shou-Hao

Chikumbo, Oliver

Chirico, Giovanni Battista

Cho, Jaeil

Choenni, Sunil

Chorley, Martin J.

Chow, Jacky

Chowdhury, Subir

Chrisinger, Benjamin

Christaras, Basile

Christen, Martin

Christophe, Sidonie

Chu, Hone-Jay

Chu, Shu-Chuan

Chybicki, Andrzej

Cinnamon, Jonathan

Ciuonzo, Domenico

Claessens, Sten

Claramunt, Christophe

Clarke, Keith

Clerc, Maurice

Clerici, Nicola

Clewley, Dan

Coccossis, Harry

Cocea, Mihaela

Codetta-Raiteri, Daniele

Coïsson, Pierdavide

Constantinou, Anthony

Coors, Volker

Cornet, Yves

Coscieme, Luca

Cox, Andrew M.

Cox, Simon

Cristiano, Piedad M.

Cui, Shiyong

Cui, Yi

Cuomo, Sabatino

Curtis, Jacqueline W.

Custódio, Danilo

Daim, Tugrul

Davis Jr., Clodoveu A.

Dawidowicz, Agnieszka

Day, Stephanie S.
De Araújo, Gustavo Medeiros

De Bruin, Sytze

De Jong, Rogier

De Meo, Pasquale

Degbelo, Auriol

Deinzer, Frank

Del Fatto, Vincenzo

Delavar, Mohmood Reza

Delfs, Jens-Olaf

Delgado, Jorge

Delikostidis, Ioannis

Delipetrev, Blagoj

Delmelle, Eric M.

Del-Val-Noguera, Elena

Demestichas, Konstantinos

Demissie, Merkebe Getachew

Deng, Yongxin

Derungs, Curdin

Deseilligny, Marc Pierrot

Devaraju, Anusuriya

Devillers, Rodolphe

Devogele, Thomas

Dewan, Ashraf

Dharssi, Imtiaz

Di Bella, Enrico

Di Martino, Ferdinando

Di Martino, Sergio

Di Zio, Simone

Dietrich, James

DiMiceli, Charlene M.

Discher, Sören

Do, $\mathrm{M}$.

Dobre, Ciprian

D'Oleire-Oltmanns, Sebastian

Dominguez Brito, Antonio

Carlos

Dominici, Donatella

Doreian, Patrick

Dos Santos, Daniel

Dougherty, Michael John

Doulamis, Nikolaos D.

Downs, Robert R.

Drummond, Mark A.

$\mathrm{Du}$, Zhiqiang

Dube, Matthew

Duchêne, Cécile

Duncan, Brean W.

Dutilleul, Pierre R.L.

Dutt-Mazumder, Aviroop

Egenhofer, M. J.

Ehrlich, Daniele

Eineder, Michael 
Eisemann, Elmar

Eldessouki, Mohamed

Elsner, Paul

Ercanoglu, Murat

Ertz, Olivier

Esposito, Christian

Fabregas, Ernesto

Facciolo, Gabriele

Fan, Hongchao

Fan, Hongchao

Fan, Jinlong

Fang, Peng

Fang, Shih-Hau

Faravelli, Lucia

Fassi, Francesco

Fearon, David

Felson, Marcus

Feng, Min

Fernández, Óscar Belmonte

Ferro, Alfredo

Filchev, Lachezar

Fischer, Andreas

Fletcher, Andrew

Fletcher, Steven J.

Flores, Gerardo

Flores-de-Santiago, Francisco

Florinsky, Igor

Forkuor, Gerald

Forootan, Ehsan

Frelich, Lee

Fricker, Geoffrey A.

$\mathrm{Fu}$, Linyun

Fuchs-Kittowski, Frank

Fujibe, Fumiaki

Fujii, Yoshiaki

Furletti, Barbara

Fuse, Takashi

Gallotti, Riccardo

Galvanin, EA Dos Santos

Galvan-Tejada, Carlos Eric

Gamo, Javier

Gao, Song

Gao, Xizhang

Garau, Chiara

Garcia, Enrique

Gemeiner, Peter

Gemitzi, Alexandra

Georgiadis, Charalampos

Gerke, Markus

Gesquière, Gilles

Gevaert, Caroline

Gharechelou, Saeid
Ghisi, Aldo

Ghorpade, Ajinkya

Gienko, Gennady

Gikas, Vassilis

Gils, Hein Van

Giovanni, Fusco

Giuliani, Gregory

Gjevestad, Jon Glenn

Gnyp, Martin L.

Goldberg, Daniel W.

Gomez, Christopher

Gómez-Gutiérrez, Álvaro

Gomez-Rubio, Virgilio

González, Alejandro

González, Higinio

Gorricho, Juan-Luis

Gorte, Ben

Goshtasby, A. Ardeshir

Grady, Sue

Granell, Carlos

Graser, Anita

Grau, Antoni

Graw, Valerie

Graziani, Alberto

Green, David R.

Green, James

Grenzdörffer, Görres

Griffin, Amy

Gröger, Gerhard

Groppe, Sven

Gruenewald, Paul

Guerra, Carlos António

Guixing, Wei

Guo, Ling-Zhong

Guo, Qinghua

Gutierrez Gallego, Jose

Antonio

H.M. Tah, Joseph

Haack, Barry

Hadas, Tomasz

Halfield, Jerry

Hall, G. Brent

Haller, Armin

Han, Weiguo

Hänsch, Ronny

Harrie, Lars

Hasan, Abdulghani

Hasan, Samiul

Hast, Anders

Hatna, Erez

Hatzichristos, Thomas

Hauthal, Eva
Haworth, James

Hazrati, Mehrnaz

He, Zhenwen

Hédl, Radim

Helbich, Marco

Henriques, R.

Hense, Andreas

Hernandez, Noelia

Herrmann, Dustin L.

Hersperger, Anna M.

Hewson, Michael

Hilari, Marc Oriol

Hills, Denise

Hilton, Brian N.

Hoarau, Charlotte

Hodgson, Michael E.

Hoel, Erik G.

Hof, Anouschka

Hofmann, Peter

Hofmann, Peter

Homayouni, Saeid

Horák, Jiří

Horner, Mark W

Horng, Shi-jinn

Hou, Dongyang

Hsiao, Rong-Shue

$\mathrm{Hsu}$, Feng Chi

$\mathrm{Hsu}$, Ting-Yu

$\mathrm{Hu}$, tangao

$\mathrm{Hu}$, Xiaogong

$\mathrm{Hu}$, Yingjie

Huang, Changchun

Huang, Chih-Yuan

Huang, Fay

Huang, R.

Huang, Shih-Chia

Huang, Shu-Wei

huang, yu

Hubert, Frédéric

Huesca, Margarita

Huettmann, Falk

Hugentobler, Urs

Hung, Ming-Chih

Hursthouse, Andrew

Hwang, Chi-Hung

Hwang, Dong-Hwan

Hwang, Hoyoung

Iantovics, Laszlo Barna

Ilia, Ioanna

Iliffe, Jonathan

Iliffe, Mark

Ishikawa, Toru 
Iso, Javier Nogueras

Iwanowski, Marcin

Iwasaki, Akira

Iyota, Taketoshi

Jacobsen, Knut Stanley

Jadidi Mardkheh, Amaneh

(Mojgan)

Jakobsson, Antti

James, Philip

Järv, Olle

Jasiewicz, JarosŁaw

Jean-François, Girres

Jen, Cheng-Lung

Jeong, Hae-Duck Joshua

Jepson, Paul

Jesús, García

Jetzek, Thorhildur

Jia, Yunyi

Jiang, Ruinian

Jiang, Shan

Jiang, Xiaoguang

Jobst, Markus

Johnson, Colin

Johnson, Peter

Jokinen, Olli

Jong, Gwo-jia

Jonietz, David

Josse, Carmen

Joyce, Karen

Jung, Jin-Kyu

K. Ramapriyan,

Hampapuram

Kada, Martin

Kadirkamanathan, Visakan

Kaim, Dominik

Kalantari, Mohsen

Kamal, Muhammad

Kamsu-Foguem, Bernard

Kang, Chaogui

Kang, Mengjun

Kaplan, Shai

Kapusta, Jozef

Karakasidis, T.E.

Karimi, Hassan

Karimi, Hassan

Kasahara, Hidekazu

Kauranne, Tuomo

Kawa, Arkadiusz

Keller, Stefan F.

Keskinarkaus, Anja

Keßler, Carsten

Khatami, Reza
Khodabandeh, Amir

Kiefer, Peter

Kim, Chang-Gil

Kim, Hyun

Kim, Jae Ik

Kim, Kyoungsook

Kim-Blanco, Paola

Kindermann, Philipp

Kinkeldey, Christoph

Kiranoudis, Chris T.

Kirste, Thomas

Klar, Jochen

Klump, Jens

Kmoch, Alexander

Ko, Connie

KO, PATRICK CHOW-IN

Kociuba, Waldemar

Kolios, S.

Kolovos, Alexander

Korbel, Piotr

Kosicki, Jakub Z.

Kostelnick, John C.

Kotthaus, Simone

Koutsias, Nikos

Koutsoudis, Anestis

Kozak, Jacek

Kraft, Angelina

Krassanakis, Vassilios

Kromer, Ryan A.

Kropatsch, Walter

Kuijpers, Bart

Kulawiak, Marcin

Kumar, Uttam

Kumar, Vimal

Kundu, Sukhamay

Kunz, Matthias

Kuo, Chung-Yen

Kupidura, Przemysław

Kuznetsov, Yuri

Kwak, Yunsik

Kwok, Ngai Ming

Lai, Sabrina

Lam, Sean Shao Wei

Lampert, Cory K.

Lampoltshammer, Thomas

Langhammer, Jakub

Lanorte, Antonio

Lasky, Ty

Lauenburg, Patrick

Law, Jane

Law, Jane

Law, Rob
Le, Hieu Hanh

Leccese, Fabio

Lee, Donghwa

Lee, Jay

Lee, Ki Yong

Lega, $\mathrm{M}$.

Leh, Mansoor D.

Leibovici, Didier

Leitner, Michael

Leon, Javier

Levin, Noam

Lewiński, Stanisław

Leyk, Stefan

$\mathrm{Li}$, Ainong

Li, Bofeng

Li, Chang

Li, Guangquan

Li, Guozhu

Li, Han

Li, Ki-Joune

Li, Ming

$\mathrm{Li}, \mathrm{Na}$

Li, Peijun

$\mathrm{Li}$, Songnian

Li, Wei

Li, Wenliang

Li, Xiang

$\mathrm{Li}$, Xiaopeng

Li, Xingxing

li, yingru

Lian, Defu

Liang, Fuyuan

Liang, Jianming

Liao, Haifeng

Liebig, Thomas

Liesenberg, Veraldo

Lieskovsky, Juraj

Ligtenberg, Arend

Lin, Feng-Cheng

Lin, Xiangguo

Lindsay, M.D.

Liu, Shufan

Liu, Xin

Liu, Xintao

Liu, Yan'an

Liu, Yu

Liu, Yun

Loibl, Wolfgang

Loke, Seng W.

Longoni, Laura

Lopes, Simone Becker

Lopez-Pellicer, Francisco J. 
López-Quílez, Antonio

Lovas, Tamás

Löwner, Marc-Oliver

Löytönen, Markku

Lu, Binbin

Lu, Yongmei

Lukeš, Petr

Luo, Wei

Lv, Zhihan

M. Dewan, Ashraf

Ma, Haiping

Ma, Lin

Ma, Mingguo

Ma, Xiaogang

Ma, Yan

Macario, Carla Geovana Do

Nascimento

Mach, Pavel

Machaj, Juraj

Maghsoudi, Yasser

Magno, Michele

Mahdavi Amiri, Ali

Maheshwari, Pankaj

Maianti, Pieralberto

Malek, Žiga

Mallinis, Giorgos

Maltese, Vincenzo

Manfreda, Salvatore

Mansberger, Reinfried

Manso Callejo, Miguel Ángel

mansourian, ali

Marcello, Javier

Marchesini, Ivan

Marcotegui, Beatriz

Mariethoz, Gregoire

Marpu, PRASHANTH

REDDY

Marsella, Maria

Martí, Pau

Martin, David

Martín, David

Martinez Dominguez,

Francisco

Martínez-Graña, Antonio

Martinez-Izquierdo, Estibaliz

Martinopoulos, George

Martins, Bruno Emanuel Da

Graça

Masiero, Andrea

Matano, Fabio

Matellán Olivera, Vicente

Mateo-Lázaro, Jesús
Mazurek, Przemysław

Mazzetti, Paolo

McGwire, Kenneth

McKenzie, Grant

Medvedev, Viktor

Meedeniya, Dulani

Meinhardt-Llopis, Enric

Melendez-Pastor, Ignacio

Membarth, Richard

Menendez, Hector

Mercorelli, Paolo

Mialon, Arnaud

Mieg, Harald A.

Mikhailova, Elena

Milanova, Mariofanna

Mirjalili, Seyedali

Mitsakou, Christina

Miura, Hiroyuki

Miyazaki, Hiroyuki

Moafipoor, Shahram

MOCCOZET, Laurent

Mocenni, Chiara

Møller-Jensen, Lasse

Moltchanova, Elena

Mondal, Md. Surabuddin

Mondino, Enrico Borgogno

Mongus, Domen

Monico, João Francisco Galera

Moore, Antoni

Moretti, Sandro

Morioka, Kazuyuki

Moshou, Dimitrios

Moulin, Bernard

Mouri, Kousuke

Moussa, Adel

Moussavi, Mahsa S.

Muche, Gerhard

Muhammad, NAWAZ

Mui, Amy

Mund, Jan-Peter

Muñoz Barreto, Jonathan

Murgante, Beniamino

Musić, Josip

Nardi, Fernando

Nardin, William

Narimah, Samat

Natarajan, Anand

Nedovic-Budic, Zorica

Negri, Rogério Galante

Neitzel, Frank

Newlands, Nathaniel K.

Newton, Andrew
Nguyen, Hoang

Nichols, David

Nilsson, Tobias

Nishio, Mayuko

Nocerino, Erica

Nourian, Pirouz

Nowak, Aleksander

Nyka, Krzysztof

O'keefe, Kyle

Okarma, Krzysztof

Oldham, Kenn

Oliveira, Sérgio C.

Olteanu-Raimond, Ana-Maria

Omitaomu, Olufemi A.

Omrani, Hichem

Orford, Scott

Ortiz, Alberto

Orus Perez, Raul

Ostendorf, Bertram

Otsuka, Yuichi

Oude Elberink, Sander

Ouled Sghaier, Moslem

Ozdemir, Ibrahim

Ozturk, Derya

Padhye, Nikhil

Painho, M.

Palaseanu-Lovejoy, Monica

Pan, Zhigang

Panagopoulos, Thomas

PANTAZIS, GEORGE

Panza, Angela

Papadimitriou, Fivos

Papaioannou, Thanasis

Papakonstantinou, Apostolos

Papakostas, George A.

Park, Byungwoon

Park, Chansik

Park, Jihye

Park, Sehyun

Parker, Chris

Parker, Christopher J.

Parmentier, Benoit

Pasolli, Edoardo

Pasolli, Edoardo

Pathan, Al Sakib Khan

Patias, Petros

Paya, Luis

Payne, Karen

Paziewski, Jacek

Perea-Ortega, José M.

Perez, Francesca

Pérez González, Pérez 
González

Pescosolido, Loreto

Peter, Michael

Peter, Ranacher

Peterson, Birgit

Petropoulos, George

Peuquet, Donna

Pingel, Thomas J.

Piras, Andrea

Pittore, Massimiliano

Pizzo, Silvio Del

Plant, Richard E.

Podobnikar, Tomaž

Polinori, Paolo

Pollino, Maurizio

Popelka, Stanislav

Popescu, Daniela

Portalés, Cristina

Potortì, Francesco

Pourabdollah, Amir

Poursanidis, Dimitris

Presotto, Andrea

Preve, Nikolaos P.

Prinz, Torsten

Pryss, Rüdiger

Pulido-Fernández, Manuel

Purves, Ross

Qian, Guoqi

Qian, Haizhong

Qin, Cheng-Zhi

Qiu, Jianbin

Quartulli, Marco

Quesnot, Teriitutea

R. García, Carmelo

Radulescu, Gheorghe M T

Rahman, Md. Shahinoor

Rahnama, Mehdi

Raissouni, Naoufal

Ramoelo, Abel

Ramondini, Massimo

Rasmussen, Jesper

Ravada, Siva

Razaque, Abdul

Razul, Azad

Rebollo-Pedruelo, Miguel

Reed, Carl

Rehrl, Karl

Reich, Siegfried

Reis, Arsénio

Renz, Matthias

Resch, Bernd

Retscher, Günther
Reyes, Melissa

Riaza, Asuncion

Ribeiro, Haroldo Valentin

Riccioli, Francesco

Richter, Kai-Florian

Ristea, Alina

Rivas, Pablo

Robertson, Colin

Rodriguez, Juan Manuel

Rodríguez, Juan Manuel

Corchado

Roggero, Marco

Romano, Emanuele

Romo Leon, Jose Raul

Roncella, Riccardo

Rosati, Luca

Roscia, Mariacristina

Rosser, Gabriel

Rotondo, Francesco

Ruckdeschel, Peter

Ruiz, Antonio Jesús Rueda

Ruiz Fernández, Luís Ángel

Rushton, Gerard

Saavedra, Mario Lillo

Sacharidis, Dimitris

Sadeghi, Morteza

Sae-Bae, Napa

Salamí, Esther

Salata, Stefano

Saldarriaga-Noreña, Hugo

Sampietro, Daniele

Sanchez-Rodriguez, David

Sandro, Bimonte

Santi, Emanuele

Santos, Celso Alberto Saibel

Sarris, Apostolos

Savelyev, Alexander

Savin, Igor

Savino, Sandro

Scalenghe, Riccardo

Schade, Sven

Scheider, Simon

Schirrmann, Michael

Scholz, Johannes

Schönauer, Robert

Schreiber, Fabio A.

Schröder, Dietrich

Schulz, Ruth

Schumacher, Jens

Scioscia, Floriano

See, Linda

Selvarajan, Sowmya
Sengupta, Raja

Şerban, Gheorghe

Serna, Andrés

Serôdio, Carlos

Serre, Damien

Sesartic, Ana

Sessa, Salvatore

Sfetsos, Athanasios

Shafri, Helmi

Shafri, Helmi

Shahabfar, Alireza

Shang, Jianga

Sharma, Bhavna

Sheeren, David

Shelestov, Andrii

Sheu, Ruey-Kai

Shi, Peijun

Shi, Xuan

Shi, Zhenwei

Shin, Yekyeong

Shortridge, Ashton

Sidiropoulos, Panagiotis

Siedentop, Stefan

Sieradzki, Rafał

Siirtola, Pekka

Silva, Luís

Silvia, Nittel

Sirmacek, Beril

Sirmacek, Beril

Sitányiová, Dana

Sithole, George

Sitzia, Tommaso

Skilodimou, Hariklia D.

Slingsby, Aidan

Snape, Jamie

Soares, João

Soden, Robert

Sofia, Giulia

Song, Houbing

Sophronides, Panayiotis

Sorokine, Alexandre

Sow, Momadou

Sparks, Ross

Spinoni, Jonathan

Stanier, Charles

Stanislawski, Lawrence

Stark, Hans-Jörg

Stefanakis, Emmanuel

Steffen, Holger

Stentoumis, Christos

Stewart, Kathleen

Stock, Kristin 
Stockhause, Martina

Strasser, Carly

Štych, Přemysl

$\mathrm{Su}$, Hongbo

$\mathrm{Su}$, Hongjun

$\mathrm{Su}$, Yanjun

$\mathrm{Su}$, Yuan-Fong

Suarez, Alvaro

Suchenwirth, Leonhard

Suen, I-Shian

Sujit, P.

Sulla-Menashe, Damien

Sun, Alexander

Sun, Danfeng

Sun, Shaohui

Sun, Yeran

Sutton, Caroline

Suzuki, Tsutomu

Świtoński, Adam

Szabó, Szilárd

Szostak-Chrzanowski, Anna

Tagg, Andy

Tahir, Ali

Takebayashi, Hideki

Takemi, Tetsuya

Tan, Kun

Tang, Shijun

Tang, Wenwu

Tang, Xinhua

Tarantino, Cristina

Tarantino, Cristina

Tarantino, Eufemia

Tayyebi, Amin

Teckentrup, Aretha

Teodoro, A. C.

Teunissen, Peter

Thapa, Rajesh Bahadur

Thappa, Rajesh Bahadur

Thinh, Nguyen Xuan

Tian, Yanshan

Tiwari, Rajesh

Tomintz, Melanie

Torrecillas, Cristina

Torres-Sospedra, Joaquin

Touya, Guillaume

Triantakonstantis, Dimitrios

Trilles, Sergio

Truong-Hong, Linh

Truong-Hong, Linh

Tsai, Sang-Bing

Tsakiri-Strati, Maria

Tsangaratos, Paraskevas
Tsela, Philemon

Tseng, Ming-Lang

Tseng, Yi-Hsing

Tsuzuki, Marcos

Tulloch, David L.

Turner, Darren

Tzavella, Katerina

Ujang, Uznir

Umek, Anton

Ureña, Manuel A.

Uuemaa, Evelyn

Valença, Jonatas

Vallet, Bruno

Valova, Iren

Van Den Eynden, Veerle

Van Der Waerden, Peter

Van Gulijk, Coen

van Prooijen, Bram Christiaan

Van Wee, Bert

Vargas, César

Vargas-Solar, Genoveva

Vaseashta, Ashok K.

Vaz, David A.

Vaz, Eric

Veganzones, Miguel Angel

Veról, Aline

Verplanke, Jeroen

Vettore, Antonio

Vieira, António

Villarreal, Miguel

Volpi, Michele

Votsi, Nefta-eleftheria P.

Wakamiya, Shoko

Wallgrün, Jan Oliver

Wan, Zhanming

Wang, Bo

Wang, Caixia

Wang, Cheng

Wang, Daisy Zhe

Wang, Hai

Wang, Hui

Wang, Huihui

Wang, Jian

Wang, Jinfeng

Wang, Sheng-Shih

Wang, Wei

Wang, Yandong

Wang, Yonghui

Ward, Timothy

Watmough, Gary

Weber, Andreas

Weber, Christiane
Wei, Xuebin

Wen, Tzai-Hung

Wheeler, Stephen

Wieland, Marc

Willenbacher, Heiko

Willers, Jeffrey

Wind, Stefan

Winter, Stephan

Wise, Nicholas

Wise, Sarah

Wojdyga, Krzysztof

Wong, David W.S.

Wong, Kwan Kit

$\mathrm{Wu}$, Changshan

$\mathrm{Wu}$, Qiusheng

Xia, Linyuan

Xie, Xingzhe

$\mathrm{Xu}$, Chen

$\mathrm{Xu}$, Peiliang

$\mathrm{Xu}$, Zengwang

Yamamoto, Toshiyuki

Yan, Dong-Ming

Yan, Wai Yeung

Yang, Bisheng

Yang, Chan-su

Yang, Chaowei

Yang, Jian

Yang, Jinn-Min

Yang, KwangSoo

Yang, Shiyu

Yao, Jing

Yao, Xiaobai

Yengoh, Genesis

Yilmazer, Nuri

Yoshiki, Syuji

Young, Derek S.

Yu, Bailang

$\mathrm{Yu}$, Chunyang

Yu, Jiangbo

Yu, Liang

Yuan, Linwang

Yuan, May

Yuan, Yihong

YUE, Yang

Yuichi, Hyakawa

Zaksek, Klemen

Zatelli, Paolo

Zecha, Stefani

Zeile, Peter

Zema, Demetrio Antonio

Zhang, Baocheng

Zhang, Bowu 
Zhang, Desheng

Zhang, Hao

Zhang, Jixian

Zhang, Kongwen

Zhang, Qingling

Zhang, Xi

Zhang, Xiaotong
Zhang, Yuanzhi

Zhao, Gang

Zhao, Junqiao

Zhao, Zhiqiang

Zhou, Huiyu

Zhou, Liang

Zhou, Meng Chu
Zhou, Xun

Zhou, Xun

Zhu, Qiang

Zhu, Yanmin

Zribi, Mehrez

Źróbek-Sokolnik, Anna

(C) 2017 by the authors; licensee MDPI, Basel, Switzerland. This article is an open access article distributed under the terms and conditions of the Creative Commons Attribution (CC-BY) license (http://creativecommons.org/licenses/by/4.0/). 